\title{
A PLATFORM FOR ENRICHING BIM REPRESENTATION THROUGH SEMANTIC WEB TECHNOLOGIES
}

\author{
Davide Simeone ${ }^{1}$, Stefano Cursi ${ }^{2}$
}

\begin{abstract}
This paper investigates the possibilities related to BIM representation enrichment through semantic web approaches, and presents a prototypal application oriented to the integration of the informative model of the building with a knowledge base developed by means of ontologies. Its scope is to enhance the semantic level of representation of building information models, as well as enlarging the representation spectrum including knowledge not directly representable in the BIM schema.
\end{abstract}

Keywords: Building Information Modelling, Semantic Enrichment, Ontologies, Building Design, BIM database.

\section{INTRODUCTION}

Since the introduction of BIM and IFC in the AEC field, the topic of semantic enrichment has been investigated in order to enhance quality and level of nongeometrical information associated to tri-dimensional representations. In this context. building information models have been presented as organised, multidisciplinary databases able to document the buildings in its different aspects (sometimes defined as dimensions of BIM) and to enhance information sharing between the different stakeholders involved in an AEC process. With the spread of BIM use in AEC, as well as the improvements of information technologies and tools to support BIM processes, the amount of information stored in BIM databases has exponentially increased, progressively showing some limits of the representation template of Building Information Modelling. In particular, two main limits can be identified: 1) the limitation of the representation spectrum and 2) the relatively poor semantic level of current building information models. In fact, they are the coherent and consistent representation of the object resulting from the design process - the building- from the perspective of its constructability. Therefore, the application domain of BIM is the one of the product or, to be more precise, of its subdomain that is the system of its physical and technological components. As a result, the main modelling entities are those representing the technological components of the building (i.e. a wall, a floor, a door), enriched with additional properties able to represent not-geometrical features of the objects (i.e. cost, construction phases, etc.). Information that cannot be formalised in those entities (for instance the one related to the normative context, or to the different contexts) are not embedded in the BIM database and, as a consequence, not integrated into the informative model of the building. In the same way, the forced perspective on the constructional description of the building, both in terms of concept/entities and

$1 \mathrm{PhD}$, Department of Civil, Construction and Environmental Engineering, Sapienza University of Rome, Italy, davide.simeone@uniroma1.it

$2 \mathrm{PhD}$, Department of Civil, Construction and Environmental Engineering, Sapienza University of Rome, Italy, stefano.cursi@uniiroma1.it 
relationships among them, reduces the possibility of providing information with its interpretative context, hindering the mutual comprehension among the different disciplines involved in a design process. To overcome these difficulties, some research has focused on the introduction of semantic web technologies to improve representation and information management in building information processes (Beetz et al. 2005, Cursi et al. 2013, Simeone et al. 2013). Such approaches rely on the use of semantic networks, systems of concepts and logical relationships (usually represented in graphs as nodes and oriented arcs) to decompose and make computable knowledge in a certain domain. In the semantic web transposition to building information modelling, part of these concepts overlaps to the family and instances elements, integrating and semantically enriching the building representation. Going a little bit deeper, AEC research in this direction has mainly focused on the use of informative ontologies (both in RDF or OWL schema) for this scope, usually referring to the Industry Foundation Classes (IFC) representational template (Pauwels et al. 2013). In this paper, we describe the development of a semantic connector (that we defined as BIM Semantic bridge) able to link a BIM database with a knowledge base generated through semantic web principles, in order to integrate and enhance the quality of semantic representation of building information models.

\section{STATE OF THE ART - BIM SEMANTIC ENRICHMENT AND SEMANTIC WEB}

The topic of semantic enrichment is relevant in AEC field because focused on improving the quality and level of non-geometric information associated with the threedimensional representations of BIM models.

In this context, the information necessary for a full comprehension of the building design, construction and management process is diversified, interrelated and, as a consequence, extremely hard to represent and manage in a single informative model. In addition to that, research has shown as sharing single information is not sufficient for real comprehension and collaboration, and it is necessary instead to provide information with its interpretative context.

In fact, while interoperability efforts and the resulted data model standard of the IFC (Hartmann et al. 2012) try to solve lexical and syntactic interoperability issues, semantic interoperability has remained to a large degree unsolved. In particular, there is a lack of research in interpreting the implicit semantics of design models, turning them to explicit facts and sharing them among the different actors involved in the AEC process.

Some research (Eastman 2014; Belsky and Sacks 2016) explored the theme of interoperability through the development of new models and methods of representation and possible evolutions of IFC-based representation schemes with the purpose downsizing the errors and reworks and improving efficiency and productivity in creating, using and reusing knowledge throughout a project lifecycle.

More recently, the introduction of the Linked Data approach has shown the potentialities of the introduction of semantic web technologies to improve representation and information management in building information processes

As well described by Pauwels (2013), the analogy between the representation schemes for the building (eg. IFC) and the description logic of semantic networks (RDF and OWL), has encouraged the creation of informative ontologies in the AEC sector, usually in combination with the IFC schemes and the Express rules, opening new possibilities for the semantic enrichment of BIM. 
In 2005, Beetz (Beetz et al. 2005) has introduced an embryonic version of the future IfcOWL, an ontology that can be considered the first step to extending the structured AEC information to the world of semantic ontologies.

In 2008 Jeong (Jeong 2008) investigated the use of ontologies for semantic sharing in multidisciplinary design. In the same period, Carrara (Carrara et al. 2009) interprets the ontology as a way to move towards knowledge-based models to improve collaboration in the AEC processes.

Such approaches rely on the use of semantic networks, systems of concepts and logical relationships (usually represented in graphs as nodes and oriented arcs) to decompose and make computable knowledge in a certain domain (Gruber 1993).

In the transposition from the semantic web to building information modelling, some of these concepts overlap the elements of families and instances, integrating and enriching the semantic representation of the building.

As briefly shown, the combination of BIM and Semantic Web it is showing gradually all its potential in enhancing the level of semantic representation in the AEC field, providing a bridge to overcome the actual gap and misalignment among the information represented in a BIM environment and those required to perform collaborative design activities.

\section{THE BIM SEMANTIC BRIDGE}

\subsection{BIM database and ontologies representation schemas: a comparison}

As stated in the introduction, the objective of this research is the development of a "semantic bridge" able to connect a BIM environment - more specifically a BIM database underlying a building information model - and a knowledge base developed through ontologies. In this way, it is possible to enrich, enhance and make more flexible the rigid, simplified semantics currently represented in building information models. A first issue in integrating these different representation systems is the problem of homogeneity of data, information and relationships between them, which are derived from different representation templates. In a BIM environment, representation of the building is is carried out in the BIM environment by relying on the triple-layered representation template made of families, family types and instances. Families are the more abstract level of the template and define classes of objects with similar meanings, functions, behaviours, and attributes. Family types are the result of specification of some common features of a set of objects belonging to the same family. Their role is to classify and formalise different variations of the same category of objects depending on the variation of features and/or attributes. the instances of elements are those that actually represent the building geometry, physicality, and configuration. In fact, the building information model of a building is generated by a process of specification that populates the model with virtual components that have unique values associated with the attributes inherited by the superordinate families/families types. In the BIM database, all the elements modelled in the BIM environment and their parameters are formalised by ID into a relational database, divided in different tables accordingly to the superordinate family.

As defined by Gruber (1993), an Ontology is "a specification of a representational vocabulary for a shared domain of discourse - definitions of classes, relations, functions, and other objects", therefore including within the same descriptive system both the concepts related to a knowledge domain and the relations among them. 
Semantic web related technologies are currently used for the creation and utilisation of ontologies. Several standard ontology editors allow description and visualisation of the entities related to a knowledge domain through the explicit definition of classes, properties, relationships and instances. Moreover, the definitions of these representational primitives include information about their meaning and constraints and their logically consistent application.

The definition of a Class includes all the declarative aspects associated with the meaning of the represented entity, even in relation to the different domains of knowledge considered. This implies that all the represented knowledge is directly related to the entity of the specific ontology, thus establishing a relational structure between all the concepts, methods, and tools of interpretation, evaluation, and control of the entity and the considered disciplinary dominion. This formalisation model is structured in a flexible, dynamic and rule-dependent way, so that, with reference to the context, and requirements, the meanings associated with the entities may be modified, or highlight knowledge base inconsistencies. A real item that fulfils the definition of a class is an "Instance" of this class.

All the descriptive and behavioural aspects related to the considered class - such as geometrical, physical and behavioural features - defined by specific values associated with the same attributes can be represented through Data Properties. The values associated with such properties can be computed by means of methods, algorithms and calculation procedures formalised and executed through inferential engines. Object Properties, instead, are used to define specific kind of relationships between two classes. An instance of an object property is a factual relation between two or more class instances.

To complete the structure of an ontology, a deductive layer consists of formalised rules able to verify and evaluate links and constraints between the entities considered in the ontology.

\subsection{The interface platform between the BIM database and the knowledge base}

Since these two representation approaches are based on different modelling principles and protocols, it was necessary to conceive a specific platform - that we defined as BIM Semantic Bridge - able to translate the two modelling environments in a homogenous format and to create correspondences between the different entities represented in them. By accessing to the two modelling databases - one underlying a BIM modelling environment, one derived from the knowledge base implemented through ontologies - the BIM Semantic Bridge performs and allows 4 main operations: 1) Reading and translation; 2) Entities mapping between the two databases in terms of classes, properties, instances, and values; 3) Comparing of the two information structures and 4) bi-directional update of the corresponding values (fig.1). 


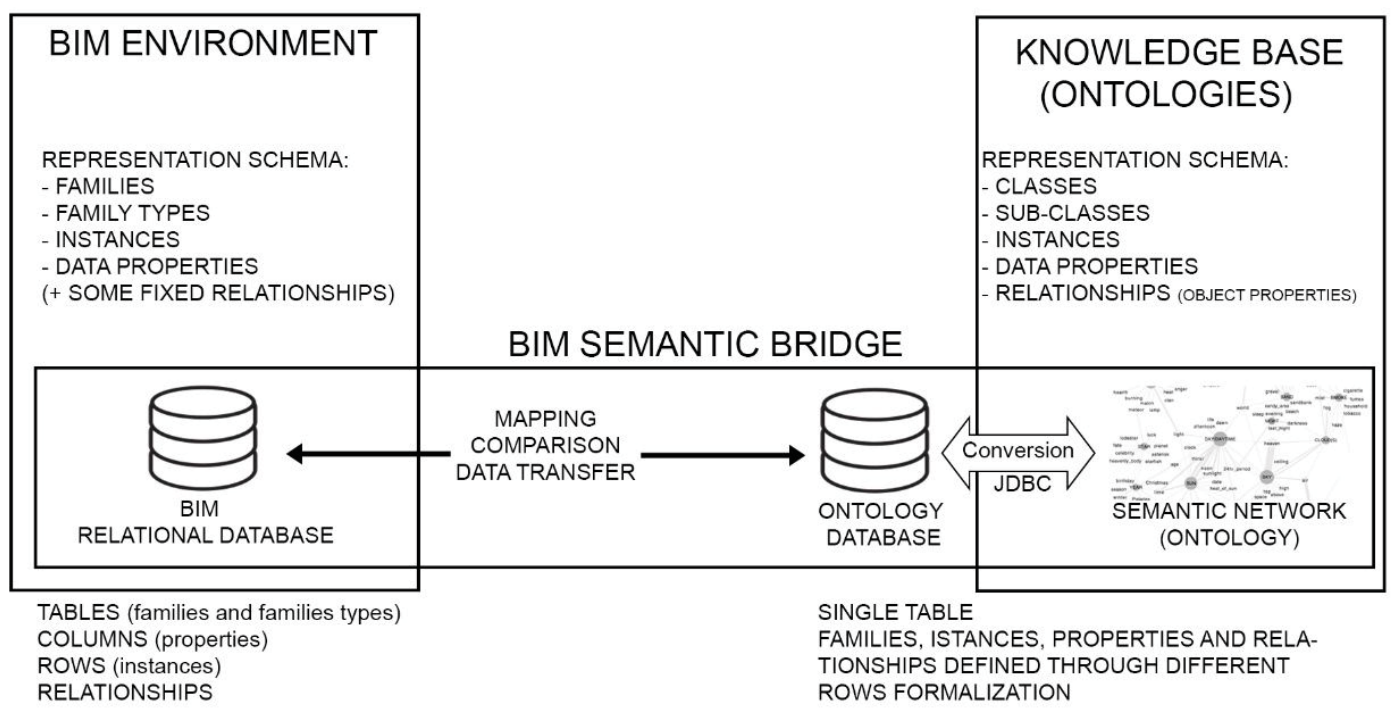

Figure 1: The conceptual schema of the BIM Semantic Bridge application.

Using the two databases as data sources, the BIM Semantic Bridge operates to reconstruct the taxonomies of classes of both sides, as well as assigned properties and derived instances. This operation homogenises the two representation allowing to generate correspondences between similar classes and data stored in both databases and perform comparison and data transfer. Current BIM models are conceived on a two layers structure family-instances while ontology-based models can be extremely flexible in the depth of the taxonomy, accordingly to the specific knowledge domain to be represented. This difference is reflected in the structures of the underlying databases: BIM databases are organised as a set of connected tables, each representing an element family with instances formalised in rows and properties in columns. In this system, it is crucial the unique ID number progressively assigned to any new instance of the model, independently from its origin family, to avoid misidentification and data overlapping. In the ontologies side, instead, databases connected to the ontology are usually made of a single table, where differences between classes, properties, relationships and instances are controlled through "type" values, and identify with a unique string made of different sub-strings referring to the "mother class", the type, etc. In this way, the ontology can be continuously extended while its structure can be easily modified during the domain formalisation. After the two databases have been imported and translated in the BIM Semantic Bridge, the system allows mapping the corresponding classes, properties, and instances between the BIM and the ontology sides. This mapping procedure is left to the user in order to take into account its requirements but, since the correspondences declarations are stored in a specific file, previous mapping schemas can be re-used in similar design processes (fig.2). 


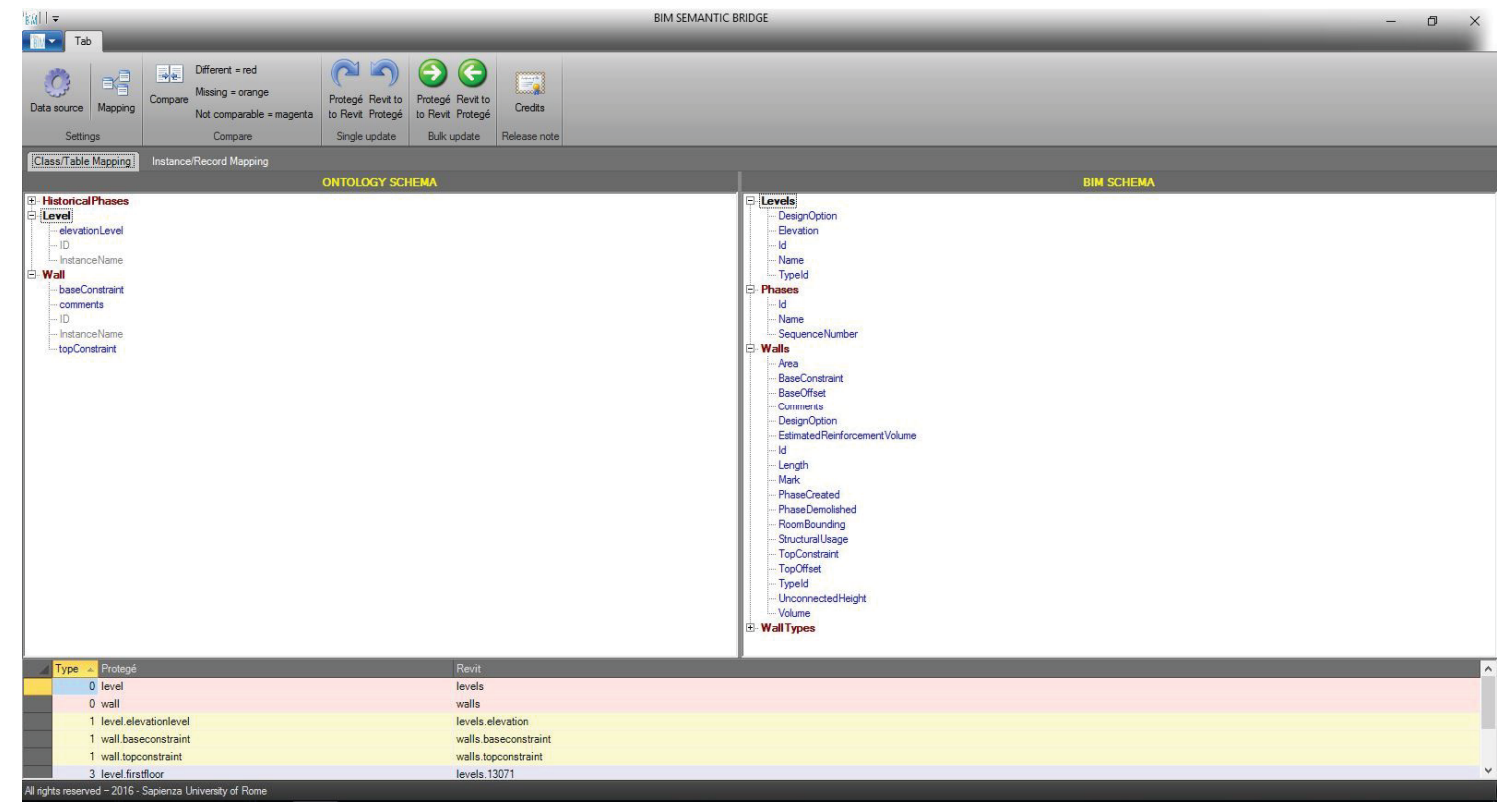

Figure 2: A screenshot from the BIM Semantic Bridge interface that shows the two areas (Ontologies on the left, BIM on the right), the mapping table (on the bottom) and the different operations that can be performed (data source definition, mapping, comparison, data transfer).

The correspondences between classes and properties are stored in an independent file that can be reused in similar projects that involved the same typologies of entities. This reduces the effort and the time necessary to formalise all the relationships between the BIM representation structure and the ontology taxonomy since a pre-formalized (but still customizable) mapping is already available. In addition, we also implemented and tested an automatic mapping feature that generates a direct correspondence between families and properties having the same name. Future developments will include other mapping procedures that take into account specific codes such as uniclass or IFC. In any case, the more relevant contribution of the proposed platform is the possibility to customise such mapping organisation, in order to integrate the BIModel with project-specific knowledge bases.

Nevertheless, the mapping of instances is still project-dependent and, at least at the current stage of research, has to be re-performed for each project. A possible way to automatize the instances mapping is currently under investigation by our research group and relies on the automatic generation of instances on both sides (with a related correspondence) when an entity is created in one of the two environments.

Once the correspondences between classes, properties, and instances have been declared, the system allows performing both operations of checking and value transfer in both directions. The first task allows detecting, for any corresponding couple of entities, differences in terms of value for any corresponding property. In addition, this comparison allows detecting all the classes, properties, and instances that have not been mapped. The second operation, instead, transfers the value stored in an entity property to the corresponding one on the other side (from the BIM DB to the Ontology DB and vice-versa), updating the corresponding database and, therefore, the related model or ontology. These operations can be performed both for datatype properties and object 
properties (namely relational properties that have as a value type, not a primitive - i.e. an integer or a string - but another instance of the model).

Regarding the prototype implementation of the BIM Semantic Bridge, it has been developed in order to connect A BIM database underlying an Autodesk Revit model and formalised through the Autodesk DBLink application and an OWL database generated through the ontology editor Protegé 3.5 and an ODBC connection.

\section{CONCLUSIONS}

This research proposed a knowledge-based system integrated with parametric objectoriented modelling platforms to improve building process and to enhance collaboration among the involved actors. The focus was on providing a framework for acquiring, structuring, representing and reusing the domain experts' knowledge and inferring new knowledge to be used during building design, construction, management and use activities.

Since its introduction in the AEC field, the BIM approach has shown both potential and limits and demonstrated only a partial suitability to complexity and necessities of building collaboration processes. This work, in particular, contributes to overcoming the low level of semantic representation allowed by the current BIM. For this aim, we presented a BIM semantic-enrichment approach that integrates BIM and semantic web methodologies, with the purpose of enhancing knowledge formalisation, sharing and management in AEC processes. To demonstrate how the proposed model can improve the performance of BIM tools using the technological paradigms of the Semantic Web, we presented its implementation through the combination of a BIM environment and a knowledge base developed through information ontologies.

A first outcome of the proposed Semantic-enriched BIM is the enhancement of the semantic level, that moves the target of the representation from information to knowledge. In addition, the proposed approach successfully extends the current BIM representation domain in order to include all the data, information, and knowledge used during an AEC process, and to make them accessible, shareable and manageable through computation. A model defined in this way can potentially both represent enough knowledge to set up and run a collaborative design process involving a number of specialists from very different specialised fields and represent the knowledge 'contained' in the final solution as the result of the design process.

The integration of the proposed platform in BIM processes, allow designers and specialists to access to larger information databases related to the project, that can embed data and knowledge not directly representable in the IM environment but still crucial for the project development. It enlarges BIM representation scopes and enhances the level of information integration and coherence in a building design process based on BIM methodologies.

This approach has made a new definition of the workflow typical of the design process; the use of the plug-ins and computer programs implemented has shown how this approach can aid the verification of design rules and constraints, demonstrating the system's overall potential.

The system we implemented attests good potential for proposing a new generation of assisted design tools, a field that permits the development of further research and analysis. 


\section{ACKNOWLEDGMENTS}

The development of the Semantic Bridge application was partially performed in conjunction with the Italian Research Project of National Interest "Bhimm. Built heritage information modelling and management. A model for architectural restoration based on knowledge". The authors want to acknowledge the work of Eng. Tommaso Asciolla in the development of the BIM Semantic Bridge implementation.

\section{REFERENCES}

Beetz, J., Van Leeuwen, J. and De Vries, B. (2005). An ontology web language notation of the industry foundation classes. Conference on Information Technology in Construction, CIB-W78, (p. 193-198). Dresden.

Belsky, M., Sacks, R. and Brilakis, I. (2016). Semantic Enrichment for Building Information Modeling. Computer-Aided Civil and Infrastructure Engineering, 31(4), 261-274.

Carrara, G., Fioravanti, A., Loffreda, G. and Trento, A. (2009). An Ontology-based Knowledge Representation Model for Cross-Disciplinary Building Design: A General Template. Computation: The New Realm of Architectural Design -27th eCAADe Conference Proceedings, (p. 367-374). Istanbul.

Cursi, S., Simeone, D. and Toldo, I. (2013). A Semantic Web Approach for Built Heritage Representation. Computer-Aided Architectural Design Futures. The Next City - New Technologies and the Future of the Built Environment - Communications in Computer and Information Science, vol. 527, pp. 383-401, Springer.

Eastman, C.M., Teicholz, P., Sacks, R. and Liston, K. (2011). BIM Handbook: A Guide to Building Information Modeling for Owners, Managers, Designers, Engineers and Contractors, 2nd ed. Wiley

Eastman, C. (2014). Knowledge-Based Building Information Modeling. In K. Kensek, \& D. Noble, Building Information Modeling: BIM in Current and Future Practice. John Wiley \& Sons Inc.

Gruber, T. (1993). A translation approach to portable ontology specifications. Knowledge Acquisition, 5(2), 199-220.

Hartmann, T., Van Meerveld, H., Vossebeld, N. and Adriaanse, A. (2012). Aligning building information model tools and construction management methods. Automation in construction, 22, 605-613.

Jeong, Y. (2008). Mediating Semantics for Multidisciplinary Collaborative Design. Berkeley: ProQuest.

Pauwels, P., Corry, E. J., Coakley, D., O'Donnell, J. and Keane, M. (2013). The Role of Linked Data and Semantic Web in Building Operation. Proceedings of the 13th annual International Conference for Enhanced Building Operations. Montréal. Addison-Wesley.

Simeone, D., Cursi S., Toldo I. and Carrara G. (2013). BIM and Knowledge Management for Building Heritage. ACADIA 14: Design Agency [Proceedings of the 34th Annual Conference of the Association for Computer Aided Design in Architecture (ACADIA), Los Angeles, pp.681-690. 